\title{
LISA \\ Laser Interferometer Space Antenna for gravitational wave measurements
}

\author{
KARSTEN DANZMANN for the LISA Study Team
}

Max-Planck-Institut für Quantenoptik, D-85748 Garching

and Universität Hannover, D-30167 Hannover, Germany

\begin{abstract}
LISA (Laser Interferometer Space Antenna) is designed to observe gravitational waves from violent events in the Universe in a frequency range from $10^{-4}$ to $10^{-1} \mathrm{~Hz}$ which is totally inaccessible to ground based experiments. It uses highly stabilised laser light ( $\mathrm{Nd}: \mathrm{YAG}, \lambda=1.064 \mu \mathrm{m}$ ) in a Michelson-type interferometer arrangement. A cluster of six spacecraft with two at each vertex of an equilateral triangle is placed in an Earth-like orbit at a distance of $1 \mathrm{AU}$ from the Sun, and $20^{\circ}$ behind the Earth. Three subsets of four adjacent spacecraft each form an interferometer comprising a central station, consisting of two relatively adjacent spacecraft ( $200 \mathrm{~km}$ apart), and two spacecraft placed at a distance of $5 \times 10^{6} \mathrm{~km}$ from the centre to form arms which make an angle of $60^{\circ}$ with each other. Each spacecraft is equipped with a laser.

A descoped LISA with only four spacecraft has undergone an ESA assessment study in the M3 cycle, and the full 6-spacecraft LISA mission has now been selected as a cornerstone in the ESA Horizon 2000-plus programme.
\end{abstract}

The LISA Assessment Report is available as ESA document SCI(94)6, May 1994.

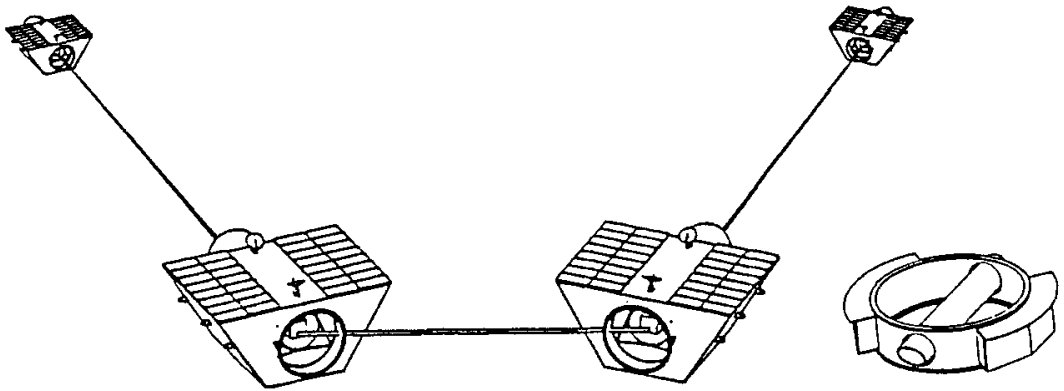

Fig. 1. Configuration of a single LISA interferometer with four spacecraft. This earlier design of trapezoidal boxes was later changed into flat circular disks, their axes normal to the interferometer plane, as indicated for a single spacecraft (with top lid removed) on the righthand side. 


\section{Overview}

The goal of LISA (Laser Interferometer Space Antenna) is to detect and study lowfrequency astrophysical gravitational radiation. The data will be used for research in astrophysics, cosmology, and fundamental physics. LISA is designed to detect the gravitational radiation from regions of the Universe that are strongly relativistic, e.g. in the vicinity of black holes. Such regions are difficult to study by conventional astronomy. The types of astrophysical sources potentially visible to LISA include galactic binaries of black holes, extragalactic supermassive black hole binaries and coalescences, and background radiation from the Big Bang. LISA will also observe galactic binary systems which are theoretically well-understood and observationally known to exist. Observation of these will provide strong verification of the instrument performance and a direct test of General Relativity.

LISA, with an array of six spacecraft, will measure such gravitational waves interferometrically. A single two-arm Michelson-type interferometer is formed from a vertex (actually consisting of two closely-spaced 'central' spacecraft), and two remote spacecraft defining the end-points of the arms, as indicated in Fig. 1. The full sixspacecraft configuration, with two spacecraft at each vertex of an equilateral triangle, thus consists of three separate, but not fully independent, interferometers. This configuration provides redundancy against component failure, gives better detection probability, and it allows the determination of polarisation.

When a gravity wave passes by it causes a strain distortion of space. LISA will detect these strains down to a level of order $10^{-23}$ in one year of observation time, by measuring the fluctuations in separation between shielded proof masses located $5 \times 10^{6} \mathrm{~km}$ apart. The measurement is performed by optical interferometry which determines the phase shift of laser light transmitted between the proof masses. Each interferometer has two symmetric arms in order to cancel out effects due to laser frequency noise. All spacecraft have a laser on board. The lasers in the two central spacecraft (which are $200 \mathrm{~km}$ apart) are phase-locked together, so they effectively behave as a single laser. The lasers in the end spacecraft are phase-locked to the incoming light, and thus act as amplifying mirrors.

Each proof mass is shielded from extraneous disturbances (e.g. solar radiation pressure) by the spacecraft in which it is accommodated. Drag-free control servos enable the spacecraft to precisely follow the proof masses. The relative displacement between the spacecraft and proof mass is measured electrostatically and the drag compensation is effected using proportional electric thrusters. Careful thermal design ensures the required mechanical stability.

\section{The need for space-based detectors}

LISA will complement the next-generation ground-based detectors (VIRGO, LIGO) by accessing the important low-frequency regime $\left(10^{-4}\right.$ to $\left.10^{-1} \mathrm{~Hz}\right)$ which will never be observable from the Earth because of terrestrial disturbances. This low-frequency window allows access to the most exciting signals, those generated by massive black hole formation and coalescences, as well as the most certain signals, such as from galactic binaries. Ground-based detectors, on the other hand, are most likely to observe the rapid bursts accompanying the final stages of a compact binary coalescence. LISA would observe the 'low-frequency' epoch where the binary systems spend most of their life. 
Cosmic background gravitational radiation, which spans a wide frequency range, may be detectable. With comparable energy sensitivites, LISA and the ground-based detectors will, in combination, provide much extended spectral coverage, essential to test cosmological models.

Extragalactic astronomy. The ideas that many galaxies (including our own) contain massive black holes, and that mergers of galaxies were common in the past, are gaining widespread acceptance. There is even evidence of binary black hole systems; an example is 3C66B which shows a precessing jet. Mergers of galaxies should produce mergers of their supermassive black holes, and their gravitational waves would be detected wherever in the Universe the event occurred. Recent calculations suggest that the event rate might even be as frequent as once per month.

The signal-to-noise ratio is typically several thousand for $10^{6} M_{\odot}$ black holes. Waves this strong might not only be useful in testing gravity, as remarked above, but may make an important contribution to fundamental cosmology. By monitoring the amplitude and phase of the merger waves while the detector rotates, both the direction and total amplitude of the waves may be determined. Then, if the direction can be used to identify the source of the waves within a known cluster of galaxies, the amplitude will give an independent distance measurement to the source. A single redshift measurement would then determine the deceleration parameter $q_{0}$, and hence the mean density of the Universe, and thus measure the total density of dark matter. Merging galaxies may also trigger the formation of massive black holes, since they may replicate conditions at the time of galaxy formation. These formation events would also be detectable and identifiable. They may also be common, even the dwarf elliptical M32 seems to have a black hole.

\section{Experiment description}

A single LISA interferometer, as shown in Fig. 2, consists of a $\mathrm{V}$ formation of four proof masses each shielded by a drag-free spacecraft. The vertex of the antenna's V-formation is formed by the two central spacecraft. In principle, one central spacecraft would be sufficient, but the optical system and attitude control requirements would be prohibitive. The four (6) spacecraft are in heliocentric orbits. They lie in a

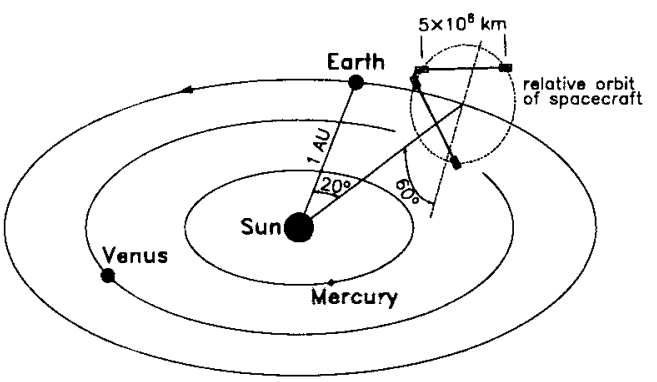

Fig. 2. Proposed LISA orbit. plane which is $60^{\circ}$ to the ecliptic such that their relative orbit is a stable circular rotation with a period of 1 year. The 'constellation' should be located as far behind the Earth as possible (but limited to about $20^{\circ}$ due to lannch vehicle constraints) to minimise Earth-induced relative velocities of the spacecraft which would lead to excessive Doppler-shifts of the transponded light. The two central spacecraft are $200 \mathrm{~km}$ apart, and the distance to the romote spacecraft, defining the interferometer arm length, is $5 \times 10^{6} \mathrm{~km}$.

The payload module consists of the inner structural carbon-epoxy cylinder with four stiffening rings, surrounded by a carbon-epoxy payload thermal shield 
cylinder. The thermal shield, cut at a $30^{\circ}$ angle at both sides, keeps sunlight from the thermally stable payload interior throughout the heliocentric orbit. The payload cylinder houses four major assemblies: the telescope assembly, the optical bench, the preamplifier disk and the radiator disk.

The telescope assembly contains a $38 \mathrm{~cm}$ diameter $\mathrm{f} / 1$ Cassegrain telescope. The primary mirror is a double-arch light-weight ultra-low expansion (ULE) design. The secondary is supported by a three-leg carbon-epoxy spider. The final quality of the plane wavefront leaving the telescope is $\lambda / 30$.

The optical bench contains the laser beam injection, detection, and beam shaping optics, and the drag-free sensor (or "accelerometer"). The proof mass of the drag-free sensor acts as the mirror at the end of the interferometer arm. The bench consists of a solid ULE plate to which all components are rigidly attached. Light from the laser is delivered to the optical bench by a single-mode fibre. About $1 \mathrm{~mW}$ is split off the $1 \mathrm{~W}$ main beam to serve as the local reference for the heterodyne measurement of the phase of the incoming beam from the far spacecraft. Also, about $1 \mathrm{~mW}$ is split off and directed towards a triangular cavity which is used as a frequency reference.

\section{Recent Developments}

A descoped LISA with only four spacecraft has been proposed for the M3 selection cycle of medium-sized missions. Of the 150 proposed missions, 7 were selected and underwent an assessment study in 1993/1994, and LISA was one of them.

A LISA mission with four spacecraft would be lost if one of the spacecraft is lost. This is also true for any mission with only one spacecraft. For small failure probabilities, the risk for LISA is in first order higher by only a factor of four. In any case, the baseline for the Cornerstone proposal assumes six spacecraft, whereby the loss of up to two spacecraft (not at the same vertex) could be tolerated without loss of the entire mission. In fact, this makes the mission more reliable than even a single spacecraft mission. Incidentally, with CLUSTER, there is a precedent for ESA to fly a multi-spacecraft missor with no backup, where the primary scientific goal wonld not be achieved in the event of a single-spacecraft failure.

The LISA M3 Assessment Study assumed four spacecraft in order to keep costs down. Using conventional technology, that mission was costed at $694 \mathrm{MAU}$. Preliminary assessments at ESTEC reveal that six spacecraft based on conventional technolgy can be accommodated in a single Ariane 5. Only three propulsion modules are required (two spacecraft per module) - not six. In combination, these factors suggest that a six-spacecraft mission need not be much more expensive than a four spacecraft mission.

More significantly, one can easily envisage that technological advances in the next 15 years will substantially reduce the mass and cost of various spacecraft and payload elements.

The full 6-spacecraft LISA misson has now been selected as a cornerstone in the ESA Horizon $2000+$ program, to be launched in the 2017 timeframe, and it has been recommended for immediate commencement of the technology development program.

The implementation of this program is subject to a small increase in the ESA science bidget starting in 2000. In the more immediate fizture, funding should be available for technical research and development of the mission concept. 